\title{
Ultrabroad-bandwidth multifrequency Raman soliton pulse trains
}

\author{
G. S. McDonald \\ Laser Optics and Spectroscopy Group, The Blackett Laboratory, \\ Imperial College of Science, Technology and Medicine, Prince Consort Road, London SW7 2BZ, UK
}

Received September 22, 1994

\begin{abstract}
I have discovered that in the coherent regime of ultrabroad-bandwidth Raman generation, a large number of long-lived soliton pulse trains are spontaneously generated. This novel solution of the dispersionless and highly transient regime, involving more than 40 distinct Raman lines of comparable amplitude, is found to be a strong attractor in the nonlinear dynamics, even when the system is initially far from this limit.
\end{abstract}

During the past three decades, stimulated Raman scattering (SRS) has been the subject of many investigations. ${ }^{1-23}$ It has been known for some time that the equations describing dispersionless twowave SRS (the interaction of the pump and first Stokes fields) have a soliton solution in the extreme transient limit. ${ }^{5}$ Initially, it was believed that an instantaneous $\pi$ phase shift had to be imposed on the input Stokes field for this soliton solution to be realized experimentally. ${ }^{6,7}$ It was then discovered that Raman solitons could arise spontaneously from vacuum phase fluctuations of the Stokes field..$^{8-11}$ Physically, the presence of a $\pi$ phase shift can reverse the Stokes gain and result in a brief temporal period of pump repletion. During this repletion period an ultrashort soliton pulse in the pump field can be generated. Experimentally, however, it turns out to be difficult to control the total number and the amplitudes of the solitons generated. This instability is partly due to a critical sensitivity of the soliton formation process on the Stokes phase shift. ${ }^{8,11}$ Any deviation of the initial phase shift from exactly $\pi$ results in unstable solitons that decay during propagation. Because these solitons strictly exist only in the extreme transient limit, their generation can be problematic because the phase modulation of the input Stokes pulse is required to be essentially instantaneous. In addition, it was proved in Ref. 12 that the conventional two-wave SRS soliton, where the pump retains a large fraction of its initial energy, cannot exist for finite energy pulses and, instead, propagates away from the main pulse and disappears.

In nearly all previous studies of SRS, higher-order Stokes and anti-Stokes waves were assumed to be of negligible amplitude. Remarkably, SRS with symmetric pumping (identical input pump and Stokes field envelopes) has only recently been examined in detail. ${ }^{13,14}$ Modeling of this regime has led to the prediction that multifrequency beams consisting of nearly 50 distinct Raman waves of comparable amplitude may be generated. Such broadband multifrequency beams may have application in inertial confinement fusion. ${ }^{16-18}$ During their generation, all the constituent waves are coupled together in nonlinear interaction, and thus the vast majority of previous analyses of SRS are no longer valid. No analytic solutions fully describing this novel broadband phenomenon are known, and systematic numerical investigations of the model equations are necessary. In this Letter I present an account of the temporal characteristics of broadband SRS and report the discovery of robust multifrequency Raman soliton pulse trains.

To model SRS, the electric field is expanded in terms of plane waves whose frequencies are given by $\omega_{n}=\omega_{0}+n \omega_{R}(n=0, \pm 1, \pm 2, \ldots)$, where $\omega_{0}$ and $\omega_{R}$ are the pump and the Stokes frequencies, respectively. We recast the governing equations into dimensionless form ${ }^{14,18,19}$ and find for the propagation of the $n$th normalized electric-field envelope, $A_{n}$, and the dynamics of the polarization wave, $P$,

$$
\begin{gathered}
\frac{\partial A_{n}}{\partial Z}=\frac{\omega_{n}}{2 \omega_{0}}\left[P^{*} A_{n+1} \exp \left(-i \gamma_{n+1} Z\right)\right. \\
\left.-P A_{n-1} \exp \left(i \gamma_{n} Z\right)\right], \\
\left(\frac{T_{2}}{t_{p}}\right) \frac{\partial P}{\partial \tau}=-P+S, \quad S=\sum_{j} A_{j} A_{j-1}^{*} \exp \left(-i \gamma_{j} Z\right) .
\end{gathered}
$$

$Z=g I_{0} z$ is the gain-length product, $g$ is the Raman gain coefficient, $I_{0}$ is the peak input intensity, $\tau$ is local time (in units of input pulse width $t_{p}$ ), and $T_{2}$ is the medium dephasing time. Dispersion gives rise to a set of finite values of normalized mistuning, $\gamma_{n}$, which can be parameterized by a single value, $\gamma_{1}$. The input fields are assumed to drive a resonant transition and, for pumping with Gaussian pulses, are taken as $A_{0}(\tau)=A_{-1}(\tau)=\exp \left(-\tau^{2}\right)$. I also consider here square input pulses, defined as $A_{0}=A_{-1}=1$ for $0 \leq \tau \leq 1$ and $A_{0}=A_{-1}=0$ for $\tau<0$ and $\tau>1$. Results are presented for rotational SRS in $\mathrm{H}_{2}$ gas pumped by the second harmonic of a Nd:YAG laser, $\omega_{R} / 2 \pi c=587 \mathrm{~cm}^{-1}$ and $\omega_{0} / 2 \pi c=18900 \mathrm{~cm}^{-1}$.

Toward the steady-state limit, $T_{2} / t_{p} \rightarrow 0$, distinct temporal regions become decoupled (incoherent) and are, ultimately, independent. A finite value of $T_{2} / t_{p}$ introduces memory effects (temporal asymmetries) 
(a)

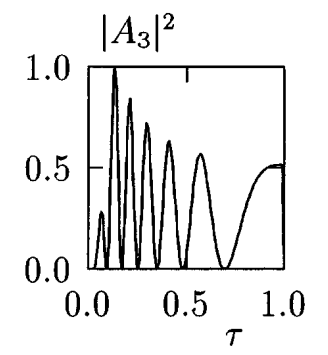

(b)

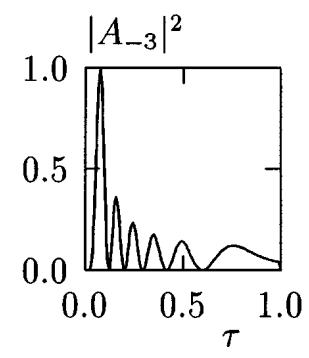

(c)

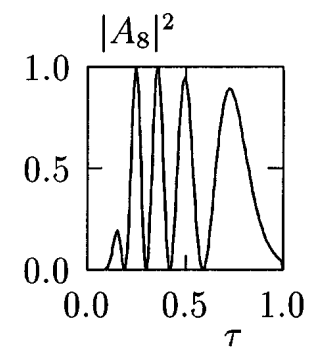

(d)

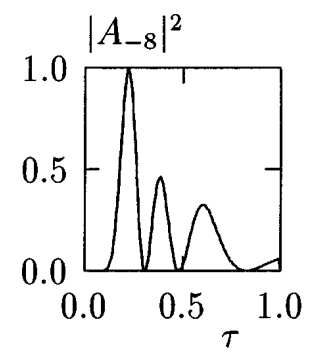

Fig. 1. Generated intensity profiles of (a) third anti-Stokes, (b) third Stokes, (c) eighth anti-Stokes, and (d) eighth Stokes waves $\left(\gamma_{1}=0, T_{2} / t_{p}=4, Z=250\right.$, and square input pulses). Each profile is plotted normalized to its peak value.

because the polarization wave is no longer constrained to follow its source term, $S$, adiabatically. The presence of both memory effects and finite dispersion can result in highly complex patterns in the time domain. ${ }^{20}$ Considering cases where $T_{2}>t_{p}$ (the coherent regime), I have discovered that, unlike in the incoherent regime, well-defined pulse trains dominate both the Stokes and the anti-Stokes orders. For square input pulses, the generated waveforms are further simplified (see Fig. 1). Each of the $\left|A_{n}(\tau)\right|^{2}$ profiles presented in this Letter is of comparable absolute intensity. A clear trend toward fewer, but wider, pulses with increasing Raman order is evident. In fully transient SRS, pulse trains are intrinsic to the nonlinear dynamics of the system and are not a simple consequence of input pulse shape. These spontaneous structures are coherent Raman soliton pulse trains, resulting from the self-organization of the interacting waves. With increasing order, either in the Stokes or the anti-Stokes direction, the wave trains are monotonically shifted in $\tau$, reflecting the continuity of the temporal redistribution of energy. Thus the underlying physical mechanism that leads to these structures is precisely the same as that which gives rise to the single soliton solution of twowave SRS.

In Fig. 2 the profiles of $|S|^{2},|P|^{2}, I_{\Sigma}=\Sigma_{j}\left|A_{j}\right|^{2}$, and bandwidth generated with square input pulses are shown. Bandwidth is defined here as the number of Stokes shifts within which the orders have comparable magnitude (within $10 \%$ of the peak intensity). Unlike in the steady-state limit, $|P|^{2}$ can grow even while $|S|^{2}$ falls rapidly to zero. $|P|$ is shown to be a relatively smooth and slowly varying mediator of the nonlinear interactions that does not itself modulate the generated waves. The cooperative nature of the formation and stabilization of these Raman soliton pulse trains is underlined by the observation that

the interacting waves collectively allow $|S|^{2} \rightarrow 0$ while $I_{\Sigma}$ remains relatively constant.

As in two-wave SRS, ${ }^{12,21,22}$ solitonic zero crossings (temporal locations where $A_{n}$ changes sign) are found to appear from large $\tau$ and stack up to form a rapid train of short pulses. Figure 3 shows the soliton pulse trains generated with Gaussian input pulses when $T_{2} / t_{p}=10$. With increasing $T_{2} / t_{p}$ it can be seen that there are fewer solitons in each pulse train. The collective characteristics of the Raman spectrum are also simple in this case (see Fig. 4). We find a smooth $|S|^{2}$ pulse that is close to temporally symmetric. The consequence of a long coherence time is to extend the lifetime of the polarization wave, which, in turn, is shown to strongly influence the profile of the bandwidth generated. In the cases presented $T_{2}$, (a)

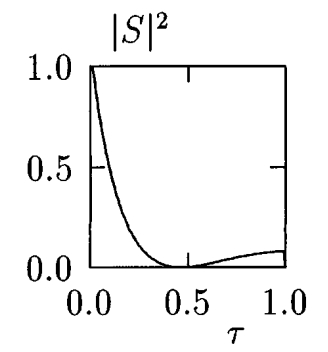

(c)

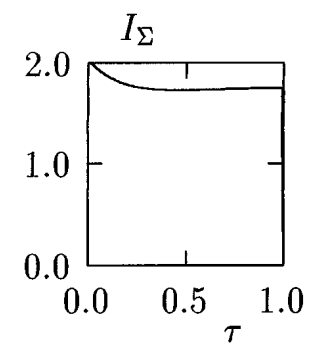

(b)

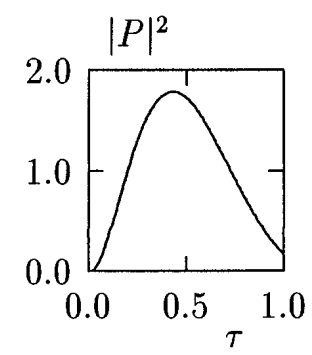

(d)

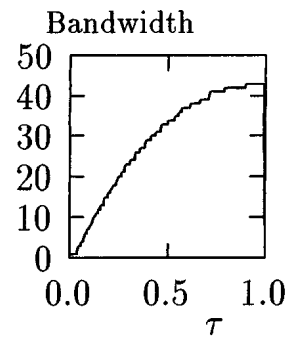

Fig. 2. Collective characteristics of the Raman soliton pulse trains of Fig. 1: (a) source term for the polarization wave, (b) magnitude of the polarization (in units of $10^{-3}$ ), (c) sum of the wave intensities, (d) bandwidth generated (in units of the Stokes shift). (a)

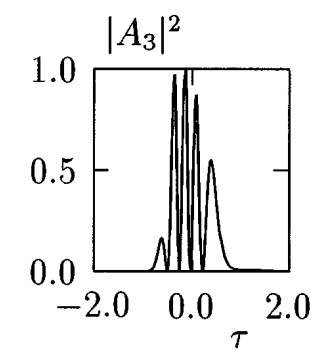

(c)

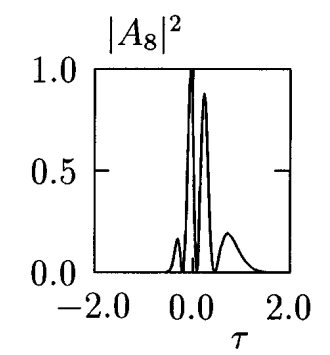

(b)

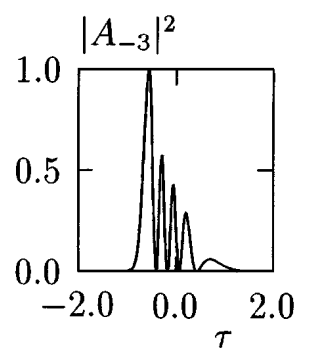

(d)

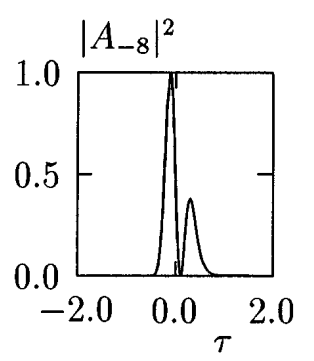

Fig. 3. As in Fig. 1, except that $T_{2} / t_{p}=10$ and Gaussian input pulses are used. 
(a)

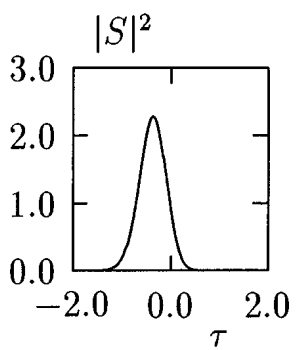

(c)

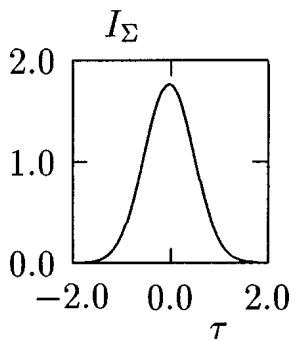

(b)

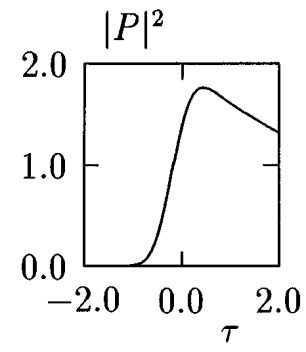

(d)

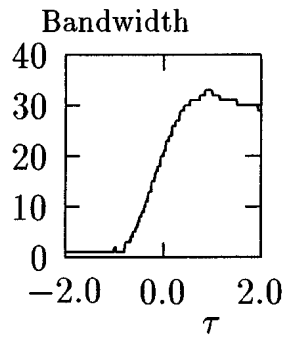

Fig. 4. As in Fig. 2, except that $T_{2} / t_{p}=10$, Gaussian input pulses are used, and $|S|^{2}$ is in units of $10^{-1}$.

in units of $t_{p}$, is 4 or 10 . Thus, contrary to previous expectations, ${ }^{16-18}$ we find that bandwidth, which in our case is ultrabroad, can be switched on in a time much less than $T_{2}$.

In a recent numerical study of three-wave $\mathrm{SRS}^{23}$ it was found that, by introduction of a $\pi$ phase shift in the input Stokes field, a single soliton can form at the anti-Stokes frequency. It was also shown that dispersive phase mismatch can lead to the decay of such solitons. We have found that, even when such phase modulation is included, the global (broadband) solution is still attracted to a set of Raman soliton pulse trains. ${ }^{20}$ Solitons of two-wave SRS have been shown to exhibit instabilities, and their formation can be triggered by small fluctuations of the Stokes field. Because solitonic zero crossings appear from large $\tau$, one might expect that the presence of noise would have a strong influence on the formation and stability of multifrequency pulse trains. We have also investigated the consequences of including complex noise in the input pump and Stokes fields and have found that soliton pulse trains are robust attractors of the nonlinear dynamics. ${ }^{20}$

In conclusion, I have discovered that Raman soliton pulse trains form spontaneously and dominate the temporal characteristics of multifrequency SRS in the coherent regime. I attribute this feature to a global SRS soliton train solution of the highly transient regime, to which the full solution is strongly attracted, even when the system is initially far from this limit. I have presented results for SRS in $\mathrm{H}_{2}$ gas, but it is expected that my overall conclusions

will have much wider applicability. In addition to considerations of other Raman media, there may be application to related phenomena such as stimulated Brillouin scattering ${ }^{1}$ and beat-wave generation of intense plasma waves. ${ }^{24}$

Useful discussions with G. H. C. New, M. J. Shaw, L. L. Losev, and A. P. Lutsenko are acknowledged. This research was supported in part by UK Science and Engineering Research Council grant GR/J04746.

\section{References}

1. Y. R. Shen and N. Bloembergen, Phys. Rev. 137, A1787 (1965).

2. C. S. Wang, Phys. Rev. 182, 482 (1969).

3. R. L. Carman, F. Shimuzu, C. S. Wang, and N. Bloembergen, Phys. Rev. A 2, 60 (1970).

4. M. E. Mack, R. L. Carman, B. Reintjes, and N. Bloembergen, Appl. Phys. Lett. 16, 209 (1970).

5. F. Y. F. Chu and A. C. Scott, Phys. Rev. A 12, 2060 (1975).

6. K. Drühl, R. G. Wenzel, and J. L. Carlsten, Phys. Rev. Lett. 51, 1171 (1983).

7. R. G. Wenzel, J. L. Carsten, and K. J. Drühl, J. Stat. Phys. 39, 621 (1985).

8. J. C. Englund and C. M. Bowden, Phys. Rev. Lett. 57, 2661 (1986).

9. J. C. Englund and C. M. Bowden, Phys. Rev. A 42, 2870 (1990).

10. D. C. MacPherson, R. C. Swanson, and J. L. Carlsten, Phys. Rev. Lett. 61, 66 (1988).

11. D. C. MacPherson, R. C. Swanson, and J. L. Carlsten, Phys. Rev. A 39, 6078 (1989).

12. C. R. Menyuk, Phys. Rev. Lett. 62, 2937 (1989).

13. L. L. Losev and A. P. Lutsenko, Kvantovaya Electron. (Moscow) 20, 1054 (1993).

14. G. S. McDonald, G. H. C. New, L. L. Losev, A. P. Lutsenko, and M. J. Shaw, Opt. Lett. 19, 1400 (1994).

15. D. Eimerl, R. S. Hargrove, and J. A. Paisner, Phys. Rev. Lett. 46, 651 (1981).

16. D. Eimerl, W. L. Kruer, and E. M. Campbell, Comments Plasma Phys. 15, 85 (1992).

17. D. Eimerl, D. Milam, and J. Yu, Phys. Rev. Lett. 70, 2738 (1993).

18. A. P. Hickman and W. K. Bischel, Phys. Rev. A 37, 2516 (1988).

19. A. P. Hickman, J. A. Paisner, and W. K. Bischel, Phys. Rev. A 33, 1788 (1986).

20. G. S. McDonald and G. H. C. New, "Coherence effects in ultrabroad bandwidth light generation," submitted to Phys. Rev. A.

21. J. N. Elgin and T. B. O'Hare, J. Phys. B 12, 159 (1979).

22. G. Hilfer and C. R. Menyuk, J. Opt. Soc. Am. B 7, 739 (1990).

23. M. Scalora, S. Singh, and C. M. Bowden, Phys. Rev. Lett. 70, 1248 (1993).

24. S. J. Karttunen and R. R. E. Salomaa, Phys. Rev. Lett. 56, 604 (1986). 